\title{
LA ESCUELA MEDIA BAJO EL MANDATO DE LA OBLIGATORIEDAD
}

\author{
Renata Giovine* \\ Liliana MarTignONi ${ }^{* *}$
}

\begin{abstract}
RESUMEN: El artículo se propone analizar cómo el mandato de la obligatoriedad escolar de la escuela media fijada por la actual ley educativa argentina pone en cuestión su histórico patrón de normalidad escolar atravesado por criterios de selección expulsiva. Para ello se abordará en primer lugar la reconstrucción de la matriz de origen de la escuela media para observar cómo se constituyó y bajo qué características dicho patrón. En segundo lugar, que estrategias políticas e institucionales se despliegan a nivel macro y micropolítico con el objetivo de introducir criterios más inclusivos, más excluyentes o una paradójica combinación de ambos. En este abordaje se encuentran presentes las oscilaciones constitutivas entre esa escuela preparatoria para el ingreso a la universidad o formadora para la inserción inmediata al mercado de trabajo. Dilema que abre el interrogante de ¿Incluir para qué? En otros términos, si incluir manteniendo esta dicotomía o si es posible pensar un modelo de escuela que prepare a la vez para ambas funciones, respetando la diversidad de los docentes, alumnos y sus familias que heterogeneizan y multiplican los sentidos de lo escolar.
\end{abstract}

Palabras clave: Escuela media. Obligatoriedad. Inclusión. Sentidos escolares.

\footnotetext{
* Doctora en Ciencias Sociales y profesora titular de la Universidad Nacional del Centro de la Provincia de Buenos Aires. E-mail: renatagiovine@gmail.com

** Doctora en Ciencias Sociales y profesora adjunta de la Universidad Nacional del Centro de la Provincia de Buenos Aires. E-mail: lmar1415@hotmail.com
} 


\section{COMPULSORY HIGH SCHOOL}

ABSTRACT: This paper examines how compulsory high school, legally established by the current education law in Argentina, questions the historical pattern of normal schooling crossed by expulsive selection criteria. It thus begins by retracing how high school emerged and which characteristics made it a standard reference. It then explores what policies and institutional strategies were deployed at the macro and micro levels in order to introduce more inclusive or exclusive criteria, or a paradoxical combination of both. This approach highlights the constitutive oscillations between high school, when preparing for university or an immediate insertion into the labor market. This dilemma raises a question: why include? In other words, should we include maintaining this dichotomy or try to think a school model that prepares for both functions, respecting the diversity of teachers, students and their families who multiply the meanings of school.

Key words: High school. Compulsory school. Inclusion. Scholar meanings.

\section{Introducción}

$I$ nnumerables investigaciones se han ocupado de evidenciar durante décadas la complejidad del nivel de educación media. Sin embargo, constituye un hecho relativamente reciente las mutaciones de sentido que lo interpelan. Un estallido que parecería estar asociado a la conjunción de diferentes cuestiones, entre las que podrían destacarse las actuales transformaciones políticas, sociales y culturales que han sacudido al mundo; relativizando y cuestionando las certezas y categorías construidas históricamente para explicar lo social: el estado nación, la clase social, la sociedad salarial, las identidades nacionales y el papel que asumió la escuela pública en relación a ellos desde la constitución de los sistemas educativos nacionales en Occidente. Los mismos en diversos países de América Latina y Europa, y particularmente en este nivel de escolarización, se encuentran hoy atravesados por una nueva racionalidad que - conjugando expansión y obligatoriedad -, pone en cuestionamiento su matriz de fundante.

En el interior de las escuelas medias se van instalando nuevos ejes de debate en la relación estado-sociedad-educación, al mismo 
tiempo que se enfrentan a problemas de diferente naturaleza. De ahí que este artículo se propone analizar cómo la política de la obligatoriedad escolar del nivel medio - recientemente fijada por la ley educativa argentina de 2006 - interpela su histórico y selectivo patrón de normalidad escolar. Cómo se pone en contradicción y conflicto aquel conjunto de artefactos y dispositivos - tanto teóricos como prácticos - que conformaron su matriz de origen al instalar - ahora y de la mano de aquella política educativa - el mandato de la inclusión. Para ello se abordará, en primer lugar la reconstrucción de la génesis y desarrollo de la escuela media en Argentina, organizada bajo la primacía de un modelo de creación elitista: el caso del Colegio Nacional Buenos Aires de 1863. Específicamente se observará cómo y bajo qué características se constituyó y permaneció dicho modelo, en tanto núcleo invariante con el transcurrir de los años, pese a algunos procesos de reforma que han ido intentando instalar desde la macropolítica una lógica más inclusiva hasta la prescripción progresiva de su obligatoriedad. En segundo lugar, el impacto que estas prescripciones tienen en el nivel micropolítico, especialmente sobre una cultura escolar como la construida en la escuela media que, caracterizada por su alta resistencia e impermeabilidad a los cambios, siente vulnerado su sentido fundacional al verse compelida a la incorporación y retención de nuevos públicos escolares. Para ello se ha recurrido a los resultados obtenidos en una investigación sobre las escuelas medias en la Provincia de Buenos Aires.

En este abordaje se hará especial hincapié en las oscilaciones constitutivas del nivel entre esa escuela preparatoria para el ingreso a la universidad o formadora de determinados conocimientos que favorezcan la inserción inmediata en el mercado de trabajo.

El mandato de origen de la escuela media argentina: elitismo y selección

La complejidad que reviste el nivel medio en América Latina y Europa encuentra en la actualidad elementos de continuidad en cuanto a los problemas que la atraviesan; producto de las transformaciones que en las últimas décadas afectan al planeta. No obstante, la comprensión del alcance de dichas mutaciones debe considerar las particularidades de los mandatos sociales y contratos fundacionales; es decir 
del contexto en que se produce la génesis y desarrollo del nivel, y que en el caso de Argentina está vinculado a su indefinición histórica en cuanto a la delimitación de su función específica. Ello es así no tanto para el caso de la formación ciudadana que se inicia desde el nivel primario, sino de aquella que surge de la tensión establecida entre un tipo de escuela preparatoria para el nivel superior y como vía inmediata de inserción laboral.

Diferentes momentos históricos atraviesan el desarrollo del nivel medio en América Latina y Argentina, cuyos elementos hegemónicos más significativos parecen entrelazarse y hasta convivir actualmente en los establecimientos de dicho nivel. Vera Godoy (1979) señala tres. El primero de ellos lo constituye la Educación Media Tradicional o de Elites que a lo largo del siglo XIX - en sus intentos de institucionalización y estructuración como nivel del sistema educativo moderno - desarrolló una modalidad impartida en colegios o liceos que se proponía, a través de una formación general, validar en el campo escolar el origen social de quienes concurrían a ella: el bachillerato. Un título que en sí mismo era la prueba suficiente o el derecho de ingreso directo a la universidad.

En Argentina, la génesis e institucionalización de la educación media estuvo asociada inicialmente al ámbito de la Provincia de Buenos Aires ${ }^{1}$ cuyos colegios - durante el siglo XIX - estuvieron bajo la tutela de la entonces provincial Universidad de Buenos Aires (creada en 1821). Posteriormente, y como producto de la constitución del estado nacional, el nivel medio ${ }^{2}$ se irá institucionalizando en base a diferentes creaciones tales como la Escuela Normal Sarmientina (1870) y el Colegio Nacional Mitrista ${ }^{3}$ (1863). Este último representa ese primer momento descripto por Vera Godoy de carácter elitista, enciclopédico, rígido, uniforme y pretendidamente homogéneo que no sólo será considerado la escuela media por antonomasia, sino que se mantendrá vigente hasta entrado el siglo Xx; constituyéndose en la matriz hegemónica de la escuela media argentina difusora de un patrón de normalidad escolar "sustrato de alta perdurabilidad en el tiempo y el espacio, estableciendo qué se entiende por escuela, por buen alumno y buen docente y que resiste a buena parte de los intentos de cambio" (Tyack y Cuban en Dussel et al., 2007, p. 13).

Sin embargo esta matriz comienza a tornarse más ambigua con el primer proceso expansivo del nivel; adquiriendo mayor complejidad 
y diversidad. Se afirma junto al bachillerato aquellas modalidades llamadas "vocacionales" (comercial y técnica) como vías menores de educación media a través de las cuales nuevos sectores sociales encontrarán un mecanismo para satisfacer sus expectativas de ascenso social. En este segundo momento, denominado por Godoy Educación Media Transicional, se va a ir evidenciando una tensión especialmente asociada a su mandato original. Es decir, la función del nivel medio como vía de ingreso a la universidad y la incorporación al mercado de empleo de funcionarios de la burocracia estatal y empleados de empresas productivas o de servicios, debilitará el poder de proporcionar "status" a quienes anteriormente se ofrecía como consolidación de su origen familiar.

A partir de este momento la educación media argentina estará imbuida de la persistente preocupación por instaurar mecanismos de selección que regulen la presión de los sectores sociales de menor nivel sociocultural, estableciendo tempranamente distintas trayectorias escolares diferenciadas por sexo y clase social. El objetivo de profesionalizar a los maestros que debían formar a los futuros ciudadanos, junto al desarrollo de otras modalidades que formasen para ocupaciones específicas de tipo manual y técnica es un reflejo de dicha preocupación. Así pueden señalarse algunas acciones que le otorgan características distintivas al nivel respecto a los otros niveles, reafirmando su temprana segmentación educativa (Braslavsky, 1985) cuyos mecanismos de selección social - a diferencia de países europeos como el caso francés - no instauró circuitos cerrados, sino más bien una "disputa abierta por las posiciones sociales y educativas [en tanto] la estructura del sistema educativo no planteaba restricciones o jerarquías en el pasaje de niveles" (Tiramonti y Ziegler, 2008 , p. 25). Las propuestas de reformas instauradas durante la primera mitad del siglo XX - desde la perspectiva de Cucuzza (1986) - estuvieron destinadas a resolver "la fractura entre proyecto educativo y proyecto de Nación procurando negar la idea de la escuela común" (p.128). Se crean diferentes escuelas para las diferentes clases sociales: salida laboral inmediata para las estratos populares y una oferta de estudios humanísticos clásicos para la elite dirigente, por un lado "los productores" y por otro "los intelectuales y dirigentes" (p. 132). ${ }^{4}$ Redes de escolarización media que el peronismo tendió a profundizar, al dejar prácticamente intacta la matriz tradicional de la escuela media e impulsar un circuito 
formativo que se adecuase a los nuevos roles ocupacionales característicos del trabajo industrial que exigían ciertos niveles técnico-profesionales y conocimientos de oficios y especialidades. La nota distintiva de las diferentes creaciones que se realizaron bajo esta política educativa es que la mayoría eran de carácter terminal y no se articulaban con el resto del sistema educativo tradicional.

Entre las décadas de 1960 y 1970 en el tercer momento descrito por Vera Godoy como Educación Media Desarrollista se profundizan los principales rasgos de la etapa anterior, pero otorgándole un sello particular producto de la Teoría del Capital Humano en boga y la introducción de las oficinas de planeamiento educativo tanto regionales como nacionales. Asimismo, se seguirá caracterizando por una notable expansión matricular de la educación media; ; destacándose en casi todos los países de la región la extinción de la Escuela Normal y - producto del incremento de la demanda para ingresar al nivel superior - la pérdida del carácter terminal con que fueron fundadas las escuelas técnicas y comerciales. En Argentina y pese al derrocamiento del peronismo, se seguirá manteniendo el impulso a ocupaciones laborales concretas, sobre todo las vinculadas al sector industrial. Ello se traduce en el incremento de la matrícula de las escuelas comercial y técnica.

Como resultado de estos tres momento puede señalarse la persistencia del carácter elitista del nivel medio y su mandato social fundante al privilegiar una formación general transformando progresivamente a todas las modalidades en "preparatorias para", ${ }^{6}$ en desmedro del carácter terminal de algunas de ellas y la creencia de que este tipo de formación favorecería la movilidad educativa y social, sobre todo a los sectores medios de la población. Esta creencia se observa en la resistencia tanto a una vocacionalización como especialización de los planes de estudio y de circuitos cerrados de formación media. De acuerdo a investigaciones educativas, ello ha producido el efecto de "secundarización" (Tiramonti, 1993).

A la vez, y en apariencia contradictoria, se observa otra tendencia: la tendencia hacia una escuela media menos segmentada, más inclusiva y progresiva (Gallart, 1984a; 1984b), al incorporar - pese a las dificultades en sostener la permanencia - a estudiantes de clase media baja e hijos de obreros calificados. ${ }^{7}$ Tendencia democratizadora que se verá 
relativizada frente a una educación media de gran complejidad - en relación al resto de los niveles - y falta de dinamismo respecto a los cambios operados en las otras esferas de la realidad social, principalmente aquellos propios de la adolescencia y juventud, además de su alta resistencia a las innovaciones organizativas, institucionales y curriculares promovidas en diferentes momentos de las políticas educativas.

Tendencias que pujan por imponerse frente a la persistencia de esa matriz de origen del nivel medio que responde al patrón de normalidad caracterizado, entre otros rasgos, por un currículum mosaico y enciclopedista, una organización institucional rígida y ritualista, una uniformidad en los procesos de enseñanza-aprendizaje y la formación de un sujeto pretendidamente homogéneo y normalizado.

Los intentos reformistas frente a la crisis del nivel medio: masificación y obligatoriedad

Con la recuperación de los regímenes democráticos en los países de la región latinoamericana, y especialmente en Argentina a partir de 1983, se reactualiza la pregunta acerca de la identidad de la escuela media, diagnosticándose su "crisis" y la necesidad de reformas. Crisis que es producto de un proceso de clausura de todo el sistema educativo, como consecuencia de las políticas autoritarias de los gobiernos militares; pero también por la persistencia de problemas que revelan la desigualdad educativa: exclusiones, retrasos, repitencia, circuitos de calidad diferenciados, etc. Podría sostenerse que se inicia un cuarto momento que llega hasta la actualidad caracterizado por producirse en un contexto de continuidad democrática aunque de constante redefinición de las políticas/reformas educativas; por transformaciones estructurales que afectan tanto a los sujetos como a las instituciones formadoras; por continuarse la fase expansiva del nivel ${ }^{8}$ y su progresiva obligatoriedad, bajo el mandato de la inclusión educativa. Desde un análisis macropolítico puede reconocerse en este último momento diferentes proyectos político-educativos.

La década de 1980 pondrá el acento en la denominada Transformación de la Educación Media (MEJ, 1989), bajo el intento de generar una reforma integral del propio nivel. Si se recuperan los rasgos 
constitutivos de aquella matriz hegemónica de la escuela media trabajados en el apartado anterior, puede observarse cómo se intenta una modificación en la propuesta curricular, siendo uno de los principales ejes "la integración pluri y multidisciplinaria ínter áreas" (p. 186); lo cual supone una nueva modalidad del trabajo escolar al crear espacios institucionales permanentes en los que se construyen estrategias para responder a diferentes problemáticas institucionales y pedagógicas. Desde lo organizacional, se propone la integración de los tres primeros años de educación media a la educación "básica, común y obligatoria" (p.127) denominado ciclo básico general, y un ciclo de orientación y especialización direccionado a la formación vocacional y laboral; intentando favorecer sobre todo la conexión de los sectores populares con el aparato productivo. En cuanto a la modalidad de trabajo docente y del aula se propone la figura del taller como un espacio de integración teórico-práctico del proceso de enseñanza-aprendizaje. Si bien esta política educativa nacional sólo se implementará de modo experimental en algunas escuelas medias nacionales, encontrará un mayor nivel de concreción en la Provincia de Buenos Aires con la llamada Reforma Dumón. ${ }^{9}$ Esta reforma intentará modificar estructuralmente todo el sistema educativo. ${ }^{10}$ En lo que respecta al nivel medio, propone ampliar la obligatoriedad escolar a los dos primeros años bajo el propósito de creación de un ciclo de orientación vocacional "procurando que el educando conozca los diversos oficios y profesiones de la vida moderna" a través de la implementación de talleres obligatorios y optativos (Plan Provincial de Gobierno, 1984-1987); primer intento de prescripción de aquel mandato a la inclusión señalada en el apartado anterior y de brindar mayores herramientas para que sea el propio alumno el que decida su trayecto escolar "científico" u "ocupacional". Con el avance de las políticas neoliberales de los años noventa, se produce un fuerte viraje en las políticas públicas estatales. Una variada literatura ha dado cuenta de cómo dichas reformas implicaron niveles significativos de exclusión social, poniendo en evidencia - como se verá más adelante - un paradójico desempeño de la educación en las nuevas políticas de transformación. ${ }^{11}$

La primera ley que regula en su conjunto al sistema educativo argentino e instrumento fundamental del Plan de Transformación Educativa (1991) ${ }^{12}$ establecerá una nueva estructura del sistema educativo, ${ }^{13}$ que en el caso de la escuela media - y de modo similar a la 
reforma Dumón - prescribe la obligatoriedad de los dos primeros años los cuales pasan a formar parte del tercer ciclo de la educación general básica (EGB3). Dicha modificación supone el desmembramiento de la escuela media que en el caso de la Provincia de Buenos Aires se anexa a la estructura organizacional y pedagógica de la escuela primaria (efecto de primarización de la EGB3). Los últimos años del nivel medio constituyen la educación polimodal que rompe con las modalidades tradicionales de escuela media, proponiendo una base general junto con una formación profesional "flexible" y "polivalente" acorde a las necesidades de un mercado más competitivo. Las nuevas modalidades ya no se vinculan a oficios y profesiones específicas, sino que responden a áreas de conocimiento disciplinar, profundizando la propuesta de la reforma de los años 80 sobre la formación tecnológica y para el trabajo. Las principales funciones continúan siendo la formación ciudadana, la formación para el mundo del trabajo y la formación para la continuación de estudios superiores. Esta última es considerada como función propedéutica del nivel y las otras dos como sus funciones específicas. Si bien esta delimitación supone una mayor claridad en el papel de la educación polimodal, no logrará romper con la creencia social en la importancia de la formación general y de corte académico. La expresión más evidente de ello es el hecho del no reconocimiento de identidad institucional que históricamente fueron construyendo las escuelas medias técnicas y artísticas, al indiferenciarlas en lo polimodal.

El componente de la matriz histórica de la escuela media que se modifica más significativamente es la tradición curricular. De acuerdo a Palamidessi (1997) y Dussel (2001), la innovación curricular apunta a la ruptura de los diseños curriculares mosaicos y su reemplazo por un formato más complejo, abierto y con posibilidades de reescritura permanente. Basados en una fundamentación didáctico-pedagógica antes que "valórica-axiomática y moralizadora", ${ }^{14}$ los contenidos disciplinarios que se elaboran se sustentan en otras lógicas argumentativas que promueven nuevas problematizaciones sobre la historia, la infancia, el lenguaje, la identidad; aunque se siga privilegiando esa visión restringida a Occidente y al capitalismo que puede limitar la proclama del respeto a la diversidad.

A diferencia de la década de los años 80, la reforma de los 90 muestra un nivel de concreción progresivo y abarcativo de todas las escuelas argentinas. Con la ampliación de la obligatoriedad escolar de 7 
a 10 años, se acentúa la ya citada tendencia a la inclusividad del nivel incorporando sectores que históricamente habían quedado excluidos de él. Numerosos estudios han mostrado la distancia existente entre su pretensión refundadora y lo que realmente fue sucediendo en el cotidiano institucional, instalándose una situación que podría señalarse como paradojal. El promovido imperativo de la inclusión contrastará con las consecuencias sociales que fue arrojando la implementación de los procesos de reforma estructural del estado, convirtiendo al sistema educativo y a la escuela en agentes mitigadores de un escenario social que arrojaría en el año 2001 a más del cincuenta por ciento de argentinos por debajo de la línea de pobreza. ${ }^{15}$

Así va cristalizándose - progresiva y persistentemente - un fenómeno de reconfiguración de sentidos que no es privativo de Argentina, sino también de otros países con diferentes niveles de desarrollo y conformación de sus sistemas educativos, ya sea latinoamericanos como europeos (Azevedo, 2000; 2001). Un cambio de sentidos que es producto de la conjunción de, por un lado la incorporación de nuevos públicos escolares a la escuela media. Por otro, producto de la crisis social fueron instalando la "contención social y afectiva" como parte de las funciones prioritarias de una escuela público-estatal que se direcciona hacia los sectores pobres de la población. Esta asociación contención-mayor cantidad de años de escolaridad convertiría a la ampliación de la obligatoriedad escolar en un dispositivo privilegiado de control social frente a una pobreza que se torna discursivamente peligrosa. Un ejemplo de ello lo constituye la siguiente justificación del Programa de Jornada Completa en el tercer ciclo de Educación General Básica (2000): "se coloca a este grupo de alumnos dentro de un marco de contenido y resguardo ante las múltiples situaciones de riesgo en que se encuentran inmersos dado las características de la etapa evolutiva en que se encuentran".

En este y otros programas y normativas va instalándose en el inicio del siglo XXI un discurso que asocia a "este grupo de alumnos" pobres - y a esta "etapa evolutiva" - los adolescentes pobres -; en el que se torna confuso diferenciar entre la prevención y la consideración punitiva de dichos sujetos, ya que se parte de la presunción de su potencialidad delictiva. Discurso que se combina con un creciente "sentimiento de inseguridad" social que se va instalando en la discusión pública con fuerza de verdad (Kessler, 2009). 
A la vez que se compele a una mayor visibilidad de las conductas y estrategias individuales por sobre las tradicionales regulaciones estatales en la definición de las trayectorias y destino de las instituciones escolares. El incremento en los niveles de inclusión y retención alcanzados por el sistema educativo - sostenidos en muchos casos por el despliegue de diferentes estrategias políticas e institucionales bajo el objetivo de garantizar la retención (Martignoni, 2002; 2008) -, convivirán con la persistencia de problemáticas vinculadas al fracaso escolar. ${ }^{16}$

La última fase de este cuarto momento se inicia a partir del año 2002, más claramente en el ámbito de la Provincia de Buenos Aires con lo que se denominó la segunda etapa de reformas que intentará distanciarse de aquellas políticas neoliberales. La sanción de la Ley $\mathrm{Na}$ cional de Educación n. 26.206/06 con el retorno a las denominaciones tradicionales de educación primaria y secundaria, cuyo rasgo más significativo será el carácter obligatorio de la totalidad de este último nivel. En relación a los componentes de la matriz que se viene trabajando a lo largo de este artículo, puede observarse la permanencia de la estructura del nivel (en ciclo básico y ciclo orientado), que si bien responde a las funciones de preparar "para" el mundo del trabajo y los estudios superiores, otorgará gran importancia a la educación técnicoprofesional observada en la creación de un circuito integrado por escuelas medias, institutos terciarios, programas y centros de formación profesional y laboral. ${ }^{17}$ Asimismo siguen vigentes los criterios de organización de contenidos de la reforma de los 90, aunque se distancia de ellos en lo referido a las modalidades que le otorgaban el carácter de "polimodal" al que ahora sería el ciclo orientado y la incorporación de los nuevos códigos culturales de los adolescentes y jóvenes. Se estaría evidenciando la intencionalidad política de reunir esos dos compartimentos estancos en los que se habían transformado la EGB3 y el Polimodal en aquellas provincias argentinas que habían optado por esta estructura organizacional y curricular, como es el caso de la Provincia de Buenos Aires como un nuevo modo de reforzar el mandato de la inclusión.

Una inclusión que desde lo discursivo vuelve a estar garantizada por el estado nacional, aunque en esta reasunción de la responsabilidad de estado en materia educativa, la educación ya no es un asunto exclusivo del sistema educativo. Más bien se va observando la formalización de la vinculación entre políticas educativas, políticas 
sociales y aquellas experiencias que propongan formatos alternativos y a veces complementarios - al escolar; los cuales no sólo se propondrán la contención social y afectiva de adolescentes y jóvenes pobres, sino que además bregarán por su inclusión en redes educativas de escolarización formal y no formal sostenidas por diferentes actores y organizaciones del estado y la sociedad civil. Aunque no puede desconocerse que el estado viene siendo desplazado social y culturalmente como principal articulador y generador de experiencias comunes.

De ahí que surge el interrogante: ¿Qué tensiones de sentido se generan entre mandatos y prácticas escolares que siguen manteniendo vivas esas oscilaciones constitutivas del nivel entre una escuela preparatoria "para" el ingreso a la universidad o escola formadora para el mundo del trabajo?

El estallido del (pretendido) sentido único de la escuela media: los nuevos públicos escolares

El recorrido por la génesis y desarrollo de la escuela media en la Argentina ha permitido observar las tensiones y cambios de sentido vinculados al cumplimiento de sus funciones. Desde aquella escuela elitista concebida para la formación de las elites con un claro propósito propedéutico de preparar para el ingreso a la universidad, hacia su progresiva masificación - promovida en parte por la creación de otras modalidades - que le otorgarán una función vinculada a la salida laboral, hasta la inclusión de nuevos sectores sociales que - plasmado en las ya citadas leyes educativas nacionales entre 1993 y 2006 - llevan a plantearse diferentes interrogantes sobre el sentido actual de la escuela media.

Estudios recientes vinculados a la política reformista de la obligatoriedad escolar ofrecen evidencias de la amalgama de sentidos que se congregan alrededor de dicha política, no sólo a partir de las determinaciones del contexto en el que la misma se diseña e implementa; sino también por la convivencia actual entre aquel mandato fundacional selectivo que fue dando forma al patrón de normalidad escolar - núcleo central de la matriz constitutiva del nivel - y el actual mandato de la inclusión social. Prescripciones y mandatos que se entrecruzan en la micropolítica escolar y que se resolverán de diferente 
modo de acuerdo al particular procesamiento que lleven a cabo cada institución y sus actores. ¿Qué expresiones encuentra esta política democratizadora de la obligatoriedad y mayor inclusión educativa en el cotidiano escolar? ¿A través de que evidencias institucionales se expresa este estallido del sentido moderno de la institución escuela? ¿Cómo se expresa este entrecruzamiento de viejos y nuevos mandatos sociales?

En primer lugar la ya señalada expansión de la escuela media, si bien ha sido acompañada por algunos cambios de tipo organizacionales, institucionales y curriculares, siguen observándose desigualdades producto de la persistencia de rasgos o componentes de la matriz hegemónica, tales como la distribución del espacio y el tiempo, los rituales y la disciplina escolar, pero sobre todo en el modelo de docente, alumno y familia esperado. En las escuelas aún persiste aquella visión clasificatoria anclada en que todo lo que se distancie de este patrón es interpretado como una desviación o anormalidad que es preciso encauzar; dando lugar a representaciones estigmatizantes que permean cada intersticio de la cultura escolar.

Los que me preocupan son los nuevos. No tenemos ni estrategias, ni armas para tratarlos: se portan mal, obviamente tiene conductas adolescentes, los compañeros les tienen miedo. Mirá, vos entrás a [primer año] y encontrás los "míos" y los "nuevos". Son dos filas completas de los míos y una fila de los nuevos: no hay forma de cruzarlos, de integrarlos debido a la edad. Son chicos de catorce y quince años, son otros físicos, otra estructura mental, otra calle, otras experiencias. El [segundo año] está dificilísimo y eso que no hay tantos nuevos. (Directora de escuela) ${ }^{18}$

"Los míos" y "los nuevos" constituyen nominaciones escolares con efectos sobre la producción de la desigualdad social. Éstas establecen líneas de demarcación que suponen distinciones más profundas que aquellas establecidas a partir de la edad, el sexo o la estructura física. Los "nuevos" son aquellos alumnos que tradicionalmente no accedían a la escuela media y a muchos de los cuales se visualiza y estigmatiza como los chicos de la calle cargados de una potencial peligrosidad que genera miedo en sus pares. Míos y nuevos que en su mayoría transitan la pobreza y la marginalidad, cuya separación y clasificación se halla atravesada más bien por el acercamiento o distanciamiento a un modelo normalizado de alumno y familia. 
En segundo lugar, se despliegan en la escuela un conjunto de estrategias políticas e institucionales que - haciendo uso en muchos casos de la negociación ${ }^{19}$ - promueven la inclusión y retención escolar; acercándola de este modo al cumplimiento de un mandato tradicionalmente asociado a la escuela primaria. Ejemplos de ellas serían la implementación de un ilimitado sistema compensatorio de evaluaciones, mayor laxitud en el cumplimiento de la asistencia a clase como en el sistema disciplinario, planes sociales que incluyen desde becas, instalación de comedores escolares, infraestructura y recursos materiales hasta la construcción de redes que - vinculando a la escuela con otras organizaciones de la sociedad civil - ofrecen a los alumnos desiguales servicios educativos y sociales. ${ }^{20}$

Por último y en tercer lugar, cabe interrogarse acerca de los sentidos que docentes, alumnos y familias de sectores pobres otorgan a la obligatoriedad de la escuela media. Los testimonios recogidos se han agrupado alrededor de dos posiciones. La primera la evalúa negativamente con argumentaciones tales como:

Una característica particular de estos alumnos es que son agresivos. O sea, el orden para ellos no existe, son espontáneos, es decir, que si se les ocurre levantarse y deambular lo hacen. La ampliación de la obligatoriedad escolar niega la capacidad de aprender a quien la tiene [el resaltado es propio]. Para los chicos éste no es un lugar para venir a aprender, es un lugar social, un lugar de encuentro. (Docente)

La escuela no les va a servir de nada... en la situación en la que estamos que no hay trabajo, para qué más años obligatorios. (Madre)

Un segundo posicionamiento agrupa los testimonios que evalúan positivamente esta política reformista, pudiendo distinguirse en su interior aquellas que consideran la obligatoriedad de la escuela media como expresión del cumplimiento de los derechos ciudadanos a la educación, quienes la entienden como una oportunidad de ingreso al mundo del trabajo, aquellos otros que la consideran como una estrategia para evitar la peligrosidad de la calle y combatir la inseguridad o alguna combinación de las anteriores.

Creo que a muchos chicos los beneficia en el sentido de que es un espacio que en determinadas horas están mal o bien, pero contenidos por nosotros. 
Muchos estarían si no es aquí, en la calle. Pero ¿cómo hago para unir esto con mi obligación de docente? (Docente)

Los que tomaron esta decisión, lo hicieron porque quizá en siete años no aprenden como en nueve años. Yo creo que tiene que haber una cantidad de años obligatorios para que todos aprendan y sepan más. (Alumna)

Tomaron esa decisión para que estemos más horas encerrados acá y no andemos haciendo cosas malas en la calle. (Alumno)

Conozco chicos que no vienen a la escuela, dejaron porque repitieron muchas veces. Los padres les han dicho que no vayan más a la escuela y se pongan a trabajar. Opino que está mal que hayan abandonado, si yo repitiera dos o tres veces seguiría igual. He hablado con ellos, les he dicho que sin estudios el día de mañana no van a tener un buen trabajo, que si tienen una familia no van a tener con qué alimentarla, o van trabajar de peón de albañil y no van a ganar nada. Pero ellos no quieren saber nada de volver a la escuela. (Alumno)

Estos testimonios dan cuenta - siguiendo a Foucault (1991) de cómo la preeminencia del objetivo estratégico dominante de un discurso gubernamental que fundamenta la importancia de ampliar la obligatoriedad escolar en tanto factor clave de desarrollo, fue mostrando con el paso del tiempo y el recrudecimiento de las condiciones socio-económicas argentinas cómo algunos de sus postulados iniciales fueron desplazándose a un segundo plano. Los altos niveles de pobreza y exclusión - con particular impacto sobre niños y adolescentes - va dando lugar a que estos elementos ejerzan un "proceso de sobredeterminación", mostrando una tensión respecto de otros, hasta llegar a evidenciar al menos una paradoja de las políticas educativas. "Contención social" y "prevención de la peligrosidad" van ocupando estratégicamente un lugar central en los discursos y adquiriendo fuerza de verdad; impactando sobre las políticas, las instituciones y la subjetividad de los actores; así como tensionando las funciones de la escuela media. Dichas funciones parecerían seguir dirimiéndose en términos dicotómicos y no con una visión superadora que piense de otro modo escuelas más inclusivas.

De ahí que las intenciones macropolíticas de inclusión deberán ser procesadas y resignificadas, no sólo como logros políticos demostrables cuantitativamente en términos de mayor matriculación y retención, sino también rompiendo con esos modelos normalizados 
de escuela, docentes, alumnos y sus familias. Así podría superarse el riesgo de que estos nuevos públicos que ingresan por vez primera a la escuela media queden sujetos a una situación de estigmatización o de no reconocimiento como sujetos diversos y portadores de derechos.

\section{Notas}

1. Cabe señalar que la ciudad de Buenos Aires, actual ciudad autónoma y capital de la República Argentina, en este momento histórico formaba parte de la provincia homónima de Buenos Aires.

2. A diferencia de la educación básica - y más allá del mandato de la Constitución Nacional de 1853/60, este nivel no contará hasta 1993 con una legislación específica que integre sus modalidades a lineamientos políticos y objetivos comunes.

3. Para un análisis de las diferencias entre los discursos fundantes de la Escuela Normal y el Colegio Nacional, véase Giovine y Huarte (2000).

4. En este proceso de regulación del ascenso social, pueden citarse la reforma Magnasco (1899 y 1900) y la propuesta Saavedra Lamas (1916) que procuraron orientar la demanda de las clases medias hacia especializaciones de tipo manual y técnica. También otros proyectos de reforma pretenderán modernizar la enseñanza media como el de Yrigoyen - Salinas (1918), Alvear - Marcó (1923), Ortiz - Coll (1939), y la reforma Astigueta (1968).

5. En el caso de Argentina, el fenómeno de la expansión en este nivel se duplica cada diez años si se toma como referencia los años 1935 en el cual la matrícula era de 104.868 alumnos; en 1945 de 201.170 alumnos; en 1955 de 471.975 alumnos y 1970 de 980.558 alumnos (Llomovatte, 1990).

6. Esta tendencia se refuerza a partir de la reforma Ortiz Coll (1939) con la implementación de los ciclos básicos para todas las modalidades de las escuelas medias.

7. En 1914 la tasa de escolarización de la población de 13 a 18 años era del 3\%. Porcentaje que - de acuerdo al censo nacional realizado 1960 - ascendió al 24,5\%.

8. Las tasas de escolarización fueron: $38,8 \%$ en 1980 , al $59,3 \%$ en 1991 y al $79,4 \%$ en 2001, según los datos aportados por los respectivos censos nacionales.

9. Resolución n. 910/84 de la Dirección General de Cultura y Educación de la Provincia de Buenos Aires.

10. Dicha reforma propone una nueva estructura conformada por el Nivel de Educación Inicial (Jardín Maternal y niños de 3 y 4 años), el Nivel de Educación Básica (niños entre los 5 años hasta los 14 años) que se constituiría en el nuevo segmento obligatorio), el Nivel de Educación Profesional (tres años del ciclo superior de la escuela media o los cuatro años correspondientes a las escuelas técnicas y agrarias) y el Nivel de Educación Superior (institutos superiores de formación docente, artística y técnica).

11. Estudios comparativos entre las reformas educativas implementadas en las dos últimas décadas, en los países del Cono Sur (Argentina, Chile, y Uruguay), muestran cómo el proceso de globalización económica y por ende las reformas estructurales al estado habrían tenido efectos en la educación al incrementar la desigualdad económica y social (Giovine, 2001). 
12. La Ley Federal de Educación n. 24.195/93 será la primera norma de alcance nacional que legisla para los diferentes sistemas educativos provinciales y la ciudad de Buenos Aires (Capital Federal); los cuales y por prescripción constitucional poseen autonomía en su definición, organización y funcionamiento.

13. La estructura del sistema educativo quedará conformada por el Nivel Inicial que incluye el jardín maternal y las salas de 3 a 5 años; convirtiendo en obligatoria esta última; el Nivel de Educación General Básica integrado por tres ciclos de tres años de duración: EGB $1\left(1^{\circ}, 2^{\circ}\right.$ y $3^{\circ}$ año), EGB2 $\left(4^{\circ}, 5^{\circ}\right.$, y $6^{\circ}$ año $)$ y EGB3 $\left(7^{\circ}\right.$ y $8^{\circ}$ y $9^{\circ}$ año; estos dos últimos del ex nivel secundario que se convertirán en obligatorios) el Nivel Polimodal de tres años de duración y el Nivel Superior integrado por las instituciones terciarias y universitarias

14. Palamidessi afirma que en los anteriores planes de estudio "habían discursos recontextualizadores morales que constituían un estrato organizador de los contenidos aún más fuerte que la graduación y lo que dictan las asignaturas. Estos discursos morales eran: un discurso sobre la armonía del universo y su sentido teleológico, progresivo; - un discurso sobre la humanidad, el hombre como ser espiritual; - un discurso sobre la patria y la nación; - un discurso sobre la democracia como forma de vida, la libertad y la solidaridad como bases de la convivencia social; - un discurso sobre la diferenciación por género; - un discurso sobre los valores humanos: lo bueno, lo bello, lo sano; - un discurso sobre el niño como ser vital en proceso de espiritualización” (1997, p. 89). Discursos que apuntaban a la formación de sujetos homogéneos.

15. De un $8,5 \%$ de personas por debajo de la línea de la pobreza en 1974 , se pasa al $24,8 \%$ en 1995 y al 32,6 \% en mayo de 2002. Momento a partir del cual trepa al 58\% en octubre de ese mismo año (INDEC, 2003).

16. De acuerdo a los datos aportados por el ministerio educativo nacional, la tasa neta de escolaridad en 2001 alcanzaba el 78,4\%. Pese a este significativo incremento, la tasa de egreso en el mismo año era del 73\% en la EGB3 y del 58,4\% en el Polimodal. Según el SITEAL, la cobertura del nivel medio creció entre 1990 y 2003 en casi 15 puntos, que para el sector más desfavorecido de la población en edad escolar ( $30 \%$ más pobre) representó un incremento de casi 20\%: del 53,1 al 73,4\%. Pero todavía siguen siendo altas la sobredad, la repitencia y el abandono, en la que se muestra una gran disparidad interjurisdiccional.

17. Ley de Educación Técnico Profesional n. 26.058/05.

18. Las entrevistas fueron realizadas entre 2007 y 2008 en escuelas públicas estatales de un distrito del centro de la Provincia de Buenos Aires.

19. La figura de la "negociación" puede tornarse positiva en la medida que ella ofrezca interesantes alternativas de desarrollo para los alumnos en cuanto a las capacidades estratégicas y de subjetivación que puedan ir incrementando sus posibilidades de alcanzar la autonomía. Pero también tornarse negativa al propiciar estigmatizaciones y descalificaciones que anulan o inhabilitan al "otro", a quien debe ser parte de una situación contractual, ya se trate de padres, alumnos y/o docentes; profundizando su situación de dependencia.

20. Estas redes se han ido formando para amortiguar la desafiliación provocada por la exclusión social. Algunas de las cuales se reconstruyeron a partir de la trayectoria que los propios alumnos de las escuelas analizadas realizaban entre la escuela y organizaciones no gubernamentales, comedores educativos y comunitarios, programas especiales de la Iglesia Católica del estado nacional y provincial, hogares de encierro y de día o de puertas abiertas (Martignoni, 2008). Algunos de los cuales serán reconocidos formalmente como por ejemplo a través de la llamada Ley Segarra, n. 13574 de 2006 (Giovine, 2010).

Cad. Cedes, Campinas, vol. 31, n. 84, p. 175-194, maio-ago. 2011

Disponível em <http://www.cedes.unicamp.br> 


\section{Referencias}

AZEVEDO, J. O ensino secundário na Europa. Lisboa: ASA, 2000.

AZEVEDO, J. Continuidades y rupturas en la enseñanza secundaria en Europa. In: Braslavsky, C. (Org.). La educación secundaria: ¡cambio o inmutabilidad?; análisis y debates de procesos europeos y latinoamericanos contemporáneos. Buenos Aires: Santillana, 2001. p. 65-109.

BRASLAVSKY, C. La discriminación educativa en Argentina. Buenos Aires: GEL, 1985.

CUCUZZA, R. El sistema educativo argentino: aportes para la discusión sobre su origen y primeras tentativas de reforma. In: HILLERT, F. et al. El sistema educativo argentino: antecedentes, formación y crisis. Buenos Aires: Cartago, 1986. p. 103-136.

DUSSEL, I. Los cambios curriculares en los ámbitos nacionales y provinciales en la Argentina (1990-2000): elementos para su análisis. Buenos Aires: Ministerio de Educación de Argentina, 2001.

DUSSEL, I. et al. Más allá de la crisis: visión de alumnos y profesores de la escuela secundaria argentina. Buenos Aires: Santillana, 2007.

FOUCAULT, M. Saber y verdad. Madrid: La Piqueta, 1991.

GALLART, M.A. La evolución de la educación secundaria 1916-1970: expansión e inmovilidad. (I) Los cambios cualitativos. Revista del Centro de Investigación y Acción Social, Buenos Aires, v. 33, n. 330, p. 2237, 1984a.

GALLART, M.A. La evolución de la educación secundaria 1916-1970. (II) El crecimiento cualitativo de la matricula y su impacto en la fuerza de trabajo". Revista del Centro de Investigación y Acción Social, Buenos Aires, v. 33, n. 331, p. 4-20, 1984 b.

GIOVINE, R. Principales aportes para el análisis de las politicas educativas y su impacto sobre la eficiencia, la eficacia y la equidad de Argentina, Chile y Uruguay en los '90. Proyecto: Alcances y resultados de las reformas educativas en Argentina, Chile y Uruguay. Buenos 
Aires: Ministerios de Educación de Argentina, Chile y Uruguay; Grupo asesor de la Universidad de Stanford; BID, 2001.

GIOVINE, R. El arte de gobernar al sistema educativo: discursos de Estado y nuevas interpelaciones. 2010. 330f. Tese (Doutorado) - FLACSO/ Sede Argentina, Buenos Aires.

GIOVINE, R.; HUARTE, G. A construção da cidadania no discurso político-educativo: Mitre e Sarmiento. Pro-Posiçôes, Campinas, v. 10, n. 2 (29), p. 26-40, jul. 1999.

KESSLER, G. El sentimiento de inseguridad. Buenos Aires: Siglo XXI, 2009.

LlOMOVATTE, S. Educación media y trabajo en Argentina. Propuesta Educativa, Buenos Aires, v. 2, n. 3-4, p. 59-84, nov. 1990.

MARTIGNONI, L. Obligatoriedad escolar y estrategias de retención en el tercer ciclo de la educación general básica: Provincia de Buenos Aires (1997-2001). 2002. 150f. Dissertação (Mestrado) - Facultad de Ciencias Humanas, Universidad Nacional del Centro de la Provincia de Buenos Aires, Tandil.

MARTIGNONI, L. Escuela y experiencias adolescentes en el gobierno de la pobreza. 2008. 424f. Tese (Doutorado) - FLACso/Sede Argentina, Buenos Aires.

PALAMIDESSI, M. Ordenes del saber y fuerzas subjetivadoras en el discurso curricular: una lectura del Programa de Educación Primaria y del Plan de Estudios del Consejo Nacional de Educación (1961). Propuesta Educativa, Buenos Aires, v. 8, n. 16, p. 86-95, 1997.

TIRAMONTI, G. El nivel medio del sistema educativo argentino. Buenos Aires: FlACSO, 1993. (Documentos e Informes de Investigación, n. 153).

TIRAMONTI G.; ZIEGLER, S. La educación de las elites: aspiraciones, estrategias y oportunidades. Buenos Aires: Paidós, 2008.

VERA GODOY, R. Disyuntivas de la educación media en América Latina. Buenos Aires: UnESCO; PNUd; OREALC, 1979. 


\section{Documentos}

Dirección General de Escuelas de la Provincia de Buenos Aires. Resolución n. 910/84.

Dirección General de Cultura y Educación de la Provincia de Buenos Aires. Programa de Jornada Completa en el tercer ciclo de Educación General Básica. La Plata, 2000.

Instituto Nacional de Estadística y Censos (INDEC): Censo Nacional de Población, Hogares y Vivienda. Años 1960-2001.

Encuesta Permanente de Hogares. Años 1975-2003.

Ministerio de Educación y Justicia de la Nación Argentina (MEJ). De los planes a la acción. La política de transformación educativa. Buenos Aires, 1989.

Ministerio de Cultura y Educación de la Nación Argentina (MCyE). Plan para la Transformación Educativa. Lineamientos políticos, ejes estratégicos y programas transformadores. Buenos Aires, 1991.

Plan Provincial de Gobierno de la Provincia de Buenos Aires, 19841987. La Plata, 1987.

SITEAL Metas educativas 2021: desafíos y oportunidades. Informes sobre tendencias sociales y educativas en América Latina. UNESCO; IIPEunEsco, Sede Regional Buenos Aires/OeI. 2010.

Recebido em 24 de março de 2011.

Aprovado em 28 de abril de 2011. 